Canadian

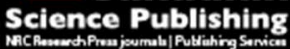

Applied Physiology, Nutrition, and Metabolism Physiologie appliquée, nutrition et métabolisme

\title{
Short-term green tea extract supplementation attenuates the postprandial blood glucose and insulin response following exercise in overweight men
}

\begin{tabular}{|r|l|}
\hline Journal: & Applied Physiology, Nutrition, and Metabolism \\
\hline Manuscript ID & apnm-2016-0169.R1 \\
\hline Manuscript Type: & Article \\
\hline Complete List of Authors: & $\begin{array}{l}\text { Martin, Brian; McMaster University, Kinesiology } \\
\text { MacInnis, Martin; McMaster University, Kinesiology } \\
\text { Gillen, Jenna; University of Michigan } \\
\text { Skelly, Lauren; McMaster University, Kinesiology } \\
\text { Gibala, Martin; McMaster University, }\end{array}$ \\
\hline Keyword: & $\begin{array}{l}\text { green tea extract, catechins, glucose metabolism, insulin, exercise } \\
\text { metabolism }\end{array}$ \\
\hline \multicolumn{2}{|c}{} \\
\hline
\end{tabular}


Short-term green tea extract supplementation attenuates the postprandial blood glucose and insulin response following exercise in overweight men.

Brian J. Martin, Martin J. MacInnis, Jenna B. Gillen, Lauren E. Skelly, and Martin J. Gibala

Department of Kinesiology, McMaster University, Hamilton, Ontario, Canada

Corresponding Author:

Martin J. Gibala

Professor and Chair

Department of Kinesiology

Ivor Wynne Centre, Rm 219

McMaster University

1280 Main St. West

Hamilton, ON L8S 4K1

Canada

905-525-9140 ext 23591

gibalam@mcmaster.ca 


\section{ABSTRACT}

Green tea extract (GTE) ingestion improves glucose homeostasis in healthy and diabetic humans, but the interactive effect of GTE and exercise is unknown. The present study examined the effect of short-term GTE supplementation on the glycemic response to an oral glucose load at rest and following an acute bout of exercise, as well as substrate oxidation during exercise. Eleven sedentary, overweight men with fasting plasma glucose $(\mathrm{FPG}) \geq 5.6 \mathrm{mmol} / \mathrm{L}(34 \pm 13 \mathrm{y}$; $\mathrm{BMI}=32 \pm 5 \mathrm{~kg} \bullet \mathrm{m}^{-2} ; \quad \mathrm{FPG}=6.8 \pm 1.0 ;$ mean $\left.\pm \mathrm{SD}\right)$ ingested $\mathrm{GTE}\left(3 \mathrm{x}^{\bullet} \mathrm{d}^{-1}, 1050 \mathrm{mg} \bullet \mathrm{d}^{-1}\right.$ total $)$ or placebo (PLA) for 7-d in a double-blind, crossover design. The effects of a $75 \mathrm{~g}$ glucose drink were assessed on 4 occasions during both GTE and PLA treatments: On days 1 and 5 at rest, and again following an acute bout of exercise on days 3 and 8. The glycemic response was assessed via an indwelling continuous glucose monitor (CGM) and venous blood draws. At rest, 1-h CGM glucose AUC was not different $(\mathrm{P}>0.05)$, but the post-exercise response was lower after GTE vs. PLA $\left(330 \pm 53\right.$ and $393 \pm 65 \mathrm{mmol} \cdot \mathrm{L}^{-1} \cdot \mathrm{min}^{-1}$, main effect of treatment, $\left.\mathrm{P}<0.05\right)$. The $1-\mathrm{h}$ postprandial peaks in venous blood glucose $\left(8.6 \pm 1.6\right.$ and $\left.9.8 \pm 2.2 \mathrm{mmol} \cdot \mathrm{L}^{-1}\right)$ and insulin $(96 \pm 59$ and $124 \pm 68 \mu \mathrm{IU} \cdot \mathrm{ml}^{-1}$ ) were also lower post-exercise with GTE vs. PLA (time $\mathrm{x}$ treatment interactions, $\mathrm{P}<0.05)$. In conclusion, short-term GTE supplementation did not affect postprandial glucose at rest; however, GTE was associated with an attenuated glycemic response following a post-exercise oral glucose load. These data suggest that GTE might alter skeletal muscle glucose uptake in humans.

\section{$\underline{\text { Key words }}$}

green tea extract; catechins; glucose metabolism; insulin; exercise metabolism 


\section{$\underline{\text { INTRODUCTION }}$}

Green tea, the unfermented leaves from the Camellia sinensis plant, is a rich source of catechins, a class of plant polyphenols that has been linked to human health and lower disease risk (Kao et al. 2006). Asian populations have used green tea medicinally for centuries, which has prompted extensive research into the health effects and its purported benefits, including antidiabetic and anti-obesity properties (Cabrera et al. 2006, Khan and Mukhtar 2007). Green tea extract (GTE) is a concentrated form of catechins and is typically high in epigallocatechin gallate (EGCG) (Cabrera et al. 2006), which accounts for $\sim 50-80 \%$ of the total catechin content in green tea and is believed to be the most biologically active molecule in GTE (Khan and Mukhtar 2007). Other major catechins include epigallocatechin, epicatechin gallate and epicatechin.

Green tea catechin (GTC) supplementation in humans has been reported to improve indices of metabolic syndrome, including insulin sensitivity, and to reduce adiposity and cholesterol (Bogdanski et al. 2012, Brown et al. 2009, Chen et al. 2009, Suliburska et al. 2012). Specifically with respect to glycemic control, two recent meta-analyses concluded that GTC ingestion lowered fasting plasma glucose (Liu et al. 2013, Zheng et al. 2013). Acute GTC administration has also been shown to improve insulin sensitivity and to reduce insulin area under the curve (AUC) during an oral glucose tolerance test (OGTT) (Venables et al. 2008). Several mechanisms have been proposed to account for the observed effect of GTC on glycemic control, based largely on cell culture models and rodent studies (Kim et al. 2016). Ueda et al. (Ueda et al. 2008) demonstrated that physiological doses of EGCG in rats (7 d of supplementation) and in L6 myotubes (15 min of treatment) increased skeletal muscle GLUT-4 translocation in both normal and insulin resistant skeletal muscle. Another study reported alterations in several glucose transporters, including GLUT-2 and GLUT-4, in various tissues 
following both acute and chronic administration of a green tea decoction or a combination of EGCG and other catechins (Snoussi et al. 2014). Lastly, three weeks of green tea consumption was shown to reduce adipose GLUT-4 translocation and concomitantly increase skeletal muscle GLUT-4 translocation (Ashida et al. 2004).

Considering the large increase in energy expenditure and the changes in glucose flux associated with exercise, the effect of GTC on glucose metabolism during exercise could be greater than that seen at rest, but this possibility has not been studied. The primary purpose of the present study was to examine the effect of short-term GTE supplementation on indices of glycemic control at rest and following exercise in response to an oral glucose load. We hypothesized that, compared to placebo (PLA), GTE would reduce the glucose and insulin response to a $75 \mathrm{~g}$ glucose beverage, at rest and following exercise. Recently, Jagannathan et al. examined various diagnostic methods used to assess the level of dysglycemia in humans, concluding that the 1-h post-load plasma glucose (PG) correlated well with the values obtained from a standard 2-h OGTT (Jagannathan et al. 2016). Thus, our aim was to use the 1-h peak glucose and insulin as a proof of principle and temporal assessment regarding length of supplementation to provide evidence for further research. A secondary purpose, given the equivocal data on the effects of GTC on fat oxidation during exercise (Dean et al. 2009, Martin et al. 2014, Randell et al. 2013, Randell et al. 2014, Richards et al. 2010, Venables et al. 2008), was to examine the potential for short-term GTE supplementation to alter whole-body energy expenditure and substrate utilization during an acute bout of exercise. We hypothesized that GTE would increase fat oxidation during an incremental exercise test designed to examine maximal rates of fat oxidation. 


\section{METHODS}

\section{Subjects}

Eleven healthy but sedentary ( $<1 \mathrm{~h}$ of physical activity/week) overweight men were recruited. Participant baseline characteristics are presented in Table 1. Participants completed a general history questionnaire to identify the presence of chronic diseases or medical conditions, medication use, as well as dietary habits, which would preclude them from participation. Participants did not habitually consume green tea beverages, supplements or medications that might influence the effects of GTE, and agreed not to ingest any such products over the course of the study. The Hamilton Integrated Research Ethics Board approved the experimental protocol, which was explained to each participant prior to obtaining written, informed consent. All procedures were performed at McMaster University.

\section{Experimental Protocol}

Overview of Study Design: A double blind, crossover design was used to compare the effects of GTE compared to a PLA. Following an initial familiarization visit, subjects performed two experimental trials in random order. Each trial involved a 7-day supplementation protocol, with measurements performed both under free-living conditions at rest, as well as during and following an acute bout of exercise. Figure 1 displays an overview of the study design. All trials were conducted in the morning, following an overnight fast. The first trial was initiated at least three days following the familiarization. This was followed by a 2 -week washout period prior to the start of the second trial.

Familiarization: During the initial visit to the laboratory, anthropometric data were collected and body composition was determined via air-displacement plethysmography BOD 
POD® (COSMED Inc., Concord, CA). Subjects then completed a graded exercise test (GXT) on a cycle ergometer (Lode Excalibur Sport V 2.0, Groningen, The Netherlands) in order to become familiar with the exercise test that was employed during the main experimental trials. The protocol involved a 5-min warm-up at 25 watts (W), after which the workload was increased by $25 \mathrm{~W}$ every 3 min until volitional fatigue to determine the maximal rate of fat oxidation (1). Heart rate (HR) (Polar Electro Oy, Kempele, Findland) and respiratory gases (Moxus modular oxygen uptake system, AEI Technologies, Pittsburgh, PA) were assessed throughout the test. Oxygen consumption $\left(\mathrm{VO}_{2}\right)$ and carbon dioxide production $\left(\mathrm{VCO}_{2}\right)$ were used in stoichiometric equations for calculation of substrate oxidation: fat oxidation $=1.695 \bullet \mathrm{VO}_{2}-1.701 \bullet \mathrm{VCO}_{2}$, and carbohydrate oxidation $=4.210 \bullet \mathrm{VCO}_{2}-2.962 \bullet \mathrm{VO}_{2}(\mathrm{Jeukendrup}$ and Wallis 2005)

Continuous Glucose Monitoring: Following familiarization and prior to each trial, subjects were fitted with a continuous glucose monitor (CGM) device (iPro ${ }^{\mathrm{TM}}$, Medtronic, Northridge, CA, USA), which provides interstitial glucose readings at 5 min intervals. The CGM was connected to a glucose sensor (Sof Sensor, Medtronic), which was inserted into the interstitial soft tissue on the anterolateral flank. Subjects were also provided with a glucose meter (OneTouch ${ }^{\circledR}$ UltraMini ${ }^{\circledR}$, Lifescan, Inc., Milpitas, CA, USA) and instructed to record capillary glucose measurements prior to each meal for CGM calibration purposes, per the manufacturer's instructions. CGM data are derived from $n=10$, owing to sensor malfunctions in one participant.

Supplementation: Subjects were provided with capsules prior to each trial that contained either decaffeinated GTE powder (Sunphenon ${ }^{\circledR}, 90 D$, Taiyo International Inc. Minneapolis MN) or PLA (cellulose). Capsules were opaque and of identical color, shape, and size to assure the contents of the GTE and PLA remained indiscernible to subjects and researchers. Given pharmacokinetic evidence that the bioavailability of ingested catechins is greater in the fasted 
state (Chow et al. 2005), and considering a half-life of $\sim 4$ h (Lee et al. 2002), subjects were instructed to ingest the capsules with $500 \mathrm{ml}$ of water $\sim 1 \mathrm{~h}$ before the glucose beverage (detailed below) or breakfast, lunch and dinner each day. Subjects ingested three capsules per day for seven consecutive days during each trial. Subjects were asked to bring their capsule bottle to each visit to assure adherence to the supplementation regimen. Each capsule in the GTE condition provided $300 \mathrm{mg}$ of catechins, the equivalent of $\sim 2-4$ cups of green tea (Henning et al. 2003). Table 2 describes the composition of the GTE used in the current study.

Experimental Trials: Subjects were tested twice at rest and twice in response to an acute bout of exercise during each trial, after varying durations of supplement ingestion. For resting measurements, subjects ingested a 75 g oral glucose beverage (Glucodex, Rougier Pharma, Mirabel, QC, Canada) under free-living conditions, in a fasted state on the first and sixth day of each intervention, after having consumed a single capsule or a total of 16 capsules, respectively. CGM data were collected and analyzed over a 1-h period immediately following glucose ingestion. For exercise measurements, subjects arrived at the laboratory in the fasted state on the third and eighth day of each trial, after consuming a total of 7 and 22 capsules, respectively. A catheter was placed into an antecubital vein for blood sampling via a collection port (BD Angiocath ${ }^{\circledR} 20 \mathrm{G}$ x 1.25 in and Q-SYTE ${ }^{\mathrm{TM}}$, Becton, Dickinson and Company, Franklin Lakes, $\mathrm{NJ})$. Blood (10 mL) was collected and placed into appropriate collection tubes (BD Vacutainer®: SST, EDTA, or Fluoride, Becton, Dickinson and Company, Franklin Lakes, NJ) for subsequent analysis. After a resting blood sample was obtained, participants performed a GXT, which was conducted as described for the familiarization visit. A second blood sample was obtained immediately following the GXT, prior to ingestion of a $75 \mathrm{~g}$ oral glucose drink. Subjects then rested for $1 \mathrm{~h}$ before a final blood sample was obtained. CGM data was again collected and 
analyzed over a 1-h period immediately following glucose ingestion.

Nutritional Controls: Subjects were provided with all meals for $48 \mathrm{~h}$ prior to the measurements made in response to the acute bouts of exercise during each trial (i.e., on the third and eighth days). Energy need was based on BOD POD ${ }^{\circledR}$ estimations $( \pm 100 \mathrm{kcal})$ for sedentary individuals, which uses the Nelson Prediction Equation (Nelson et al. 1992). The meals were individualized for each subject and provided 2,262 $\pm 216 \mathrm{kcal}^{\circ} \mathrm{day}^{-1}$ (protein $=2.5 \pm 0.3$, carbohydrate $=5.4 \pm 0.7$, and fat $=1.6 \pm 0.1, \mathrm{~g} \mathrm{~kg}^{-1}$ ). Subjects were also instructed to refrain from physical activity and alcohol during the periods of controlled nutrition intake. Lastly, subjects completed a general questionnaire to identify nutrition, supplementation, and medication patterns that might influence the effects of the GTE.

Blood Analyses: Blood collected for serum (insulin) was allowed to clot for $30 \mathrm{~min}$ after collection then separated by centrifugation $(10 \mathrm{~min}$ at $1,300 \mathrm{~g})$ and stored at $-80{ }^{\circ} \mathrm{C}$ for later analysis. Plasma obtained from either EDTA (glycerol and free fatty acids (FFA)) or fluoride (glucose) tubes were immediately centrifuged $(10 \mathrm{~min}$ at $1,300 \mathrm{~g})$ and stored at $-80{ }^{\circ} \mathrm{C}$ for later analysis. Plasma glucose was analyzed using a glucose (hexokinase) reagent kit (Pointe Scientific, Canton MI), and serum insulin was measured through an ELISA (ALPCO Diagnostics, Salem NH). Plasma glycerol (Sigma-Aldrich CO. LLC. St. Louis, MO) and plasma FFA (WAKO Diagnostics, Richmond, VA) were determined with enzymatic colorimetric assays.

\section{Statistical Analyses}

A 3-way repeated measures ANOVA (time $\mathrm{x}$ day $\mathrm{x}$ treatment) was used to analyze venous blood data. All blood analyses were conducted in duplicate with coefficient of variations of: 8.2, 4.5, 7.8, and $1.4 \%$ for glucose, insulin, FFA, and glycerol, respectively. A 2-way repeated measures ANOVA (day x treatment) was used for all CGM data and exercise variables 
( $\mathrm{HR}, \mathrm{VO}_{2}, \mathrm{VCO}_{2}$, fat and carbohydrate oxidation). Significant interactions were analyzed using pairwise comparisons for simple interactions. All assumptions were met and significance was accepted with a $\mathrm{P}<0.05$ (2-tailed). All data were analyzed using Statistical Package for Social Sciences (SPSS, version 20.0, Inc, Chicago, IL).

\section{RESULTS}

There were no day $\mathrm{x}$ treatment interactions for any variable; thus, all data reported are the means \pm SD across the two days of each experimental trial for all variables.

\section{Resting Measures}

There was no effect of GTE on glucose AUC in response to the $75 \mathrm{~g}$ glucose drink, when ingested under fasted, resting conditions $(\mathrm{GTE}=394 \pm 70$ vs. PLA $=409 \pm 78, \mathrm{mmol} / \mathrm{L} \bullet$ 60 min, $\mathrm{P}=0.51$ ). Fasting, pre-exercise blood glucose, insulin, glycerol and FFA concentrations were also not different with GTE vs. PLA $(\mathrm{P}>0.05$, Table 3$)$. The homeostatic model assessment of insulin resistance (HOMA-IR) was also not different between treatments $(\mathrm{GTE}=$ $3.8 \pm 3.4$ vs. $4.6 \pm 2.8, \mathrm{P}=0.21)$

\section{Exercise}

Peak oxygen uptake during the GXT was not different between treatments $(\mathrm{GTE}=24.2$ \pm 4.2 vs. $\left.\mathrm{PLA}=23.7 \pm 5.0 \mathrm{~mL} \bullet \mathrm{kg}^{-1} \bullet \mathrm{min}^{-1}, \mathrm{P}=0.43\right)$. Peak fat oxidation was similar with GTE

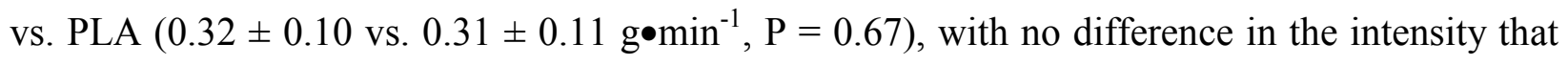
elicited peak fat oxidation $(\mathrm{GTE}=45 \pm 25$ and PLA $=45 \pm 18 \mathrm{~W}, \mathrm{P}=1.0)$. Similarly, there were no differences in the mean carbohydrate oxidation during the GXT with GTE vs. PLA (1.36 \pm 0.21 vs. $1.35 \pm 0.29 \mathrm{~g} \bullet \mathrm{min}^{-1} \mathrm{P}=0.83$ ). Supplementary Figure $\mathrm{S} 1$, displays the fat and carbohydrate oxidation rates at each workload during the GXT, which is truncated to the highest 
workload that all participants achieved. Lastly, mean exercise heart rate did not differ between

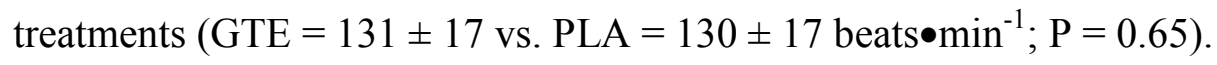

\section{Post-exercise}

Immediately following exercise, blood glucose, insulin, glycerol and FFA concentrations were not different $(\mathrm{P}>0.05$, Table 3$)$. In response to the $75 \mathrm{~g}$ glucose beverage during exercise recovery, the 1-h glucose AUC from the CGM was lower with GTE vs. PLA (330 \pm 53 vs. $393 \pm$ $65 \mathrm{mmol} / \mathrm{L} \bullet 60 \mathrm{~min}$, main effect of treatment, $\mathrm{P}<0.001$; Fig. 2). Peak blood glucose and insulin were both lower with GTE vs. PLA (time $\mathrm{x}$ treatment interactions, $\mathrm{P}=0.04$ and 0.01 , respectively; Fig. 3). Lastly, 1-h post-exercise glycerol and FFA concentrations were not different between treatments $(\mathrm{P}>0.05$, Table 3$)$.

\section{DISCUSSION}

The major novel finding from this study was that GTE attenuated the glucose and insulin response to an oral glucose load following acute exercise but had no effect on these variables under resting conditions. GTE or PLA was administered 3x/day over a 7-day period with assessments made after 2 and 7 days of supplementation. For each trial, we examined the effects of GTE on the glycemic response to oral glucose ingestion at rest and during exercise recovery. GTE attenuated the postprandial rise in glucose and insulin following exercise, but the effect on day 7 was similar to that on day 2. Thus, the duration of short-term GTE supplementation did not influence the magnitude of the response. Notably, we did not observe any effects of GTE under resting conditions, and the effect of GTE was only apparent when combined with an exercise stimulus. 
While we are the first to report an effect of GTE on blood glucose and insulin postexercise, GTE was previously shown to acutely improve insulin sensitivity and the insulinemic response following an OGTT at rest. Venables et al. (Venables et al. 2008) provided participants with three doses (dose $=340 \mathrm{mg}$ polyphenols, $136 \mathrm{mg}$ EGCG) of GTE over $24 \mathrm{~h}$, with the last dose at least $1 \mathrm{~h}$ prior to the OGTT, which lowered insulin AUC and increased insulin sensitivity relative to PLA. Similarly, a green tea beverage ( $1.5 \mathrm{~g}$ green tea powder), administered $10 \mathrm{~min}$ prior to an OGTT lowered glucose levels at 30 and 120 min post glucose ingestion vs. PLA (Tsuneki et al. 2004). The effects of green tea have also been observed following chronic supplementation. A recent study that investigated the effects of a high polyphenol diet over 8 weeks, supplemented partially by green tea, observed a significant reduction in post-load glucose AUC and improved insulin sensitivity (Bozzetto et al. 2015). In contrast, 8 weeks of EGCG supplementation (400 mg, 2x/day), with the last dose taken the evening prior to an OGTT, did not improve insulin sensitivity or lower glucose when compared to a PLA (Brown et al. 2009). Two notable differences between these studies, aside from the duration of the treatments, is the treatment itself (GTE vs. EGCG) and the timing of the last dose. Firstly, although EGCG is the most bioactive of the four main catechins, it is potentially more effective when ingested as a component with other catechins, such as with GTE, than when ingested alone (Bode and Dong 2009). Secondly, the half-life of tea catechins in the blood is very short ( 4 h) (Lee et al. 2002), and thus the timing of the last ingested dose between these studies is a potential reason for the differences. As we also did not report an effect of GTE at rest, even with a catechin-rich GTE supplement and short duration of time between the final dose and the measurement, the differences among studies could indicate a relatively small effect size of GTE on resting glucose metabolism. 
The lower blood glucose and insulin concentrations observed following glucose ingestion during recovery from exercise in the present study is suggestive of either decreased glucose absorption, enhanced glucose uptake from the blood, or both. Randomized controlled trials have shown lower fasting blood glucose and glycated hemoglobin (HbA1c) from GTC supplementation and green tea consumption over several weeks to months (Liu et al. 2013, Zheng et al. 2013). However, a $1-\mathrm{h} P G \geq 8.6 \mathrm{mmol} / \mathrm{L}$ following ingestion of a 75 glucose beverage was recently identified as a better identifier of high-risk individuals than HbAlc (Jagannathan et al. 2016), further supporting to the relevant nature of the findings from the present study. Although insight into potential mechanisms for these effects is only available from rodent models, one putative mechanism observed following GTC treatment is an increased translocation of glucose transporters. For example, GLUT-4 translocation increased in rat skeletal muscle following 7 days of supplementation as well as in L6 myotubes following an acute 15-min treatment (24). EGCG has also been shown to reverse fatty acid induced impairments in the insulin-signaling pathway (Li et al. 2011), which improved downstream translocation of GLUT-4 in skeletal muscle and adipose tissue. These mechanisms, if also present in humans, would explain the lower insulin AUC observed by Venables et al. (Venables et al. 2008) as well as the data from the current study. Interestingly, others have shown that EGCG increases activation of 5'-AMP-activated protein kinase (AMPK) (Santana et al. 2015), which is a target of the common diabetic medication, Metformin. GTC might also improve insulin sensitivity through reductions in ROS-generating inflammatory markers (Yan et al. 2012). Overall, several mechanisms have been put forward to explain the ability of GTC to influence glycemic control in humans, either at rest or combined with exercise, but more mechanistic studies of humans are needed. 
There is evidence of metabolic and physiologic effects of GTC during exercise in humans, which could have contributed to the differences we observed on glucose and insulin during exercise recovery. It has been shown that GTC increased fat oxidation during exercise, improved $\mathrm{VO}_{2} \max$, lowered steady-state exercise $\mathrm{HR}$, and increased markers of lipolysis (Martin et al. 2014, Randell et al. 2013, Richards et al. 2010, Venables and Jeukendrup 2008). The most often-cited mechanism by which GTC might exert these effects during exercise is through inhibition of catechol-O-methyl transferase (COMT), an enzyme responsible for the degradation of catecholamines, such as epinephrine (EPI) and norepinephrine (NE) (Dulloo et al. 1999, Lu et al. 2003). Indeed, GTC are purported to increase adrenergic drive through increased betareceptor stimulation. However, we recently reported no effect of GTE on either EPI or NE following $48 \mathrm{~h}$ of GTE supplementation, when compared to a PLA. (Martin et al. 2014). Additionally, Hodgson et al. (Hodgson et al. 2013) observed no differences in metabolites indicative of increases in catecholamines following 1 or 7 days of GTE administration. Considering that we concurrently examined the effects of GTE on exercise substrate use and markers of fat catabolism (FFA and glycerol), neither of which were different between treatments, our data do not support an effect of GTE on fuel use in exercising humans. Consequently, it is unlikely that an interaction between GTE supplementation and the exercise stimulus on substrate use contributed to the changes observed in glucose or insulin during the post-exercise period.

There are limitations to the present study that should be considered. First, using CGM under free-living conditions instead of more direct measurements of blood glucose and insulin might have limited our ability to observe an effect of GTE on the response to the oral glucose beverage at rest. Although CGM data is clinically accurate (Radermecker et al. 2013), and it was 
a novel means of examining the effects of GTE under free-living conditions, it does not allow us to make conclusions on the effects of GTE on insulin at rest, and it is not completely equivalent to performing an OGTT with venous blood draws. Secondly, while the post-exercise data is strengthened by similar glucose responses measured with CGM and in blood, these data only allow us to conclude that glucose and insulin concentrations were lower with GTE; however, these data do not provide mechanistic insight to explain these results. Specifically, we do not have data to demonstrate whether GTE alters glucose absorption or uptake. Lastly, while these data are applicable to sedentary, overweight men, there is limited data on the effects of GTE in a female population. Thus, possible sex-based differences might exist with regard to exercise substrate oxidation and glycemic control in a female population.

The data presented in this study suggest that GTE could have positive health benefits. Given the common practice of eating in the post-exercise period, consuming GTE regularly might enhance glucose uptake and/or reduce spikes in glucose and insulin concentrations, which would have implications for both active populations, as well as those with impaired glycemic control. For example, this effect would be especially advantageous in overweight populations beginning an exercise routine, where the normal postprandial response increases insulin and reduces lipolysis and potentially post-exercise fat oxidation (Horowitz et al. 1997). Interestingly, one study previously showed that GTE reduced respiratory exchange ratio at rest, but only in the postprandial period (Boschmann and Thielecke 2007). As we are the first to test the effects of GTE on postprandial glucose responses following exercise, more research is needed to confirm our results and to identify the physiological mechanism(s) involved.

In summary, the present study found that 2 or $7 \mathrm{~d}$ of GTE supplementation attenuated the glucose and insulin responses to an oral glucose load following acute exercise. We did not 
observe any effects of GTE on glucose metabolism under resting conditions or whole-body substrate utilization during exercise. Our findings, along with previous evidence showing that GTE improves insulin sensitivity in humans (Venables et al. 2008) and alters glucose transporters in rodents (Ueda et al. 2008), suggest potential metabolic effects of GTE that warrant further investigation. 


\section{Conflict of Interest}

The authors declare no conflicts of interest

Acknowledgements

The GTE powder was an in-kind contribution provided by TAIYO International, Inc.

B.J.M., J.B.G., L.E.S. and M.J.G. designed research; B.J.M., J.B.G. and L.E.S. conducted

research; B.J.M., M.J.M., and M.J.G. analyzed data; B.J.M., M.J.M. and M.J.G wrote the paper;

M.J.G. had primary responsibility for final content; all authors read and approved the final manuscript. 


\section{REFERENCES}

Ashida H, Furuyashiki T, Nagayasu H, Bessho H, Sakakibara H, Hashimoto T, Kanazawa K. 2004. Anti-obesity actions of green tea: possible involvements in modulation of the glucose uptake system and suppression of the adipogenesis-related transcription factors. Biofactors. 22(14):135-140.

Bode AM, Dong Z. 2009. Epigallocatechin 3-gallate and green tea catechins: United they work, divided they fail. Cancer Prev Res (Phila). 2(6):514-517.

Bogdanski P, Suliburska J, Szulinska M, Stepien M, Pupek-Musialik D, Jablecka A. 2012. Green tea extract reduces blood pressure, inflammatory biomarkers, and oxidative stress and improves parameters associated with insulin resistance in obese, hypertensive patients. Nutr Res. 32(6):421-427.

Boschmann M, Thielecke F. 2007. The effects of epigallocatechin-3-gallate on thermogenesis and fat oxidation in obese men: a pilot study. J Am Coll Nutr. 26(4):389S-395S.

Bozzetto L, Annuzzi G, Pacini G, Costabile G, Vetrani C, Vitale M, Griffo E, Giacco A, De Natale C, Cocozza S, et al. 2015. Polyphenol-rich diets improve glucose metabolism in people at high cardiometabolic risk: a controlled randomised intervention trial. Diabetologia. 58(7):15511560.

Brown AL, Lane J, Coverly J, Stocks J, Jackson S, Stephen A, Bluck L, Coward A, Hendrickx H. 2009. Effects of dietary supplementation with the green tea polyphenol epigallocatechin-3gallate on insulin resistance and associated metabolic risk factors: randomized controlled trial. $\mathrm{Br}$ J Nutr. 101(6):886-894.

Cabrera C, Artacho R, Gimenez R. 2006. Beneficial effects of green tea--a review. J Am Coll Nutr. 25(2):79-99. 
Chen N, Bezzina R, Hinch E, Lewandowski PA, Cameron-Smith D, Mathai ML, Jois M, Sinclair AJ, Begg DP, Wark JD, et al. 2009. Green tea, black tea, and epigallocatechin modify body composition, improve glucose tolerance, and differentially alter metabolic gene expression in rats fed a high-fat diet. Nutr Res. 29(11):784-793.

Chow HH, Hakim IA, Vining DR, Crowell JA, Ranger-Moore J, Chew WM, Celaya CA, Rodney SR, Hara Y, Alberts DS. 2005. Effects of dosing condition on the oral bioavailability of green tea catechins after single-dose administration of Polyphenon E in healthy individuals. Clin Cancer Res. 11(12):4627-4633.

Dean S, Braakhuis A, Paton C. 2009. The effects of EGCG on fat oxidation and endurance performance in male cyclists. Int J Sport Nutr Exerc Metab. 19(6):624-644.

Dulloo AG, Duret C, Rohrer D, Girardier L, Mensi N, Fathi M, Chantre P, Vandermander J. 1999. Efficacy of a green tea extract rich in catechin polyphenols and caffeine in increasing 24-h energy expenditure and fat oxidation in humans. Am J Clin Nutr. 70(6):1040-1045.

Henning SM, Fajardo-Lira C, Lee HW, Youssefian AA, Go VL, Heber D. 2003. Catechin content of 18 teas and a green tea extract supplement correlates with the antioxidant capacity. Nutr Cancer. 45(2):226-235.

Hodgson AB, Randell RK, Boon N, Garczarek U, Mela DJ, Jeukendrup AE, Jacobs DM. 2013. Metabolic response to green tea extract during rest and moderate-intensity exercise. J Nutr Biochem. 24(1):325-334.

Horowitz JF, Mora-Rodriguez R, Byerley LO, Coyle EF. 1997. Lipolytic suppression following carbohydrate ingestion limits fat oxidation during exercise. Am J Physiol. 273(4 Pt 1):E768-775. 
Jagannathan R, Sevick MA, Fink D, Dankner R, Chetrit A, Roth J, Buysschaert M, Bergman M. 2016. The 1-hour post-load glucose level is more effective than HbA1c for screening dysglycemia. Acta Diabetol. DOI 10.1007/s00592-015-0829-6

Jeukendrup AE, Wallis GA. 2005. Measurement of substrate oxidation during exercise by means of gas exchange measurements. Int J Sports Med. 26 Suppl 1:S28-37.

Kao YH, Chang HH, Lee MJ, Chen CL. 2006. Tea, obesity, and diabetes. Mol Nutr Food Res. 50(2):188-210.

Khan N, Mukhtar H. 2007. Tea polyphenols for health promotion. Life Sci. 81(7):519-533.

Kim Y, Keogh JB, Clifton PM. 2016. Polyphenols and Glycemic Control. Nutrients. 8(1).

Lee MJ, Maliakal P, Chen L, Meng X, Bondoc FY, Prabhu S, Lambert G, Mohr S, Yang CS. 2002. Pharmacokinetics of tea catechins after ingestion of green tea and (-)-epigallocatechin-3gallate by humans: formation of different metabolites and individual variability. Cancer Epidemiol Biomarkers Prev. 11(10 Pt 1):1025-1032.

Li Y, Zhao S, Zhang W, Zhao P, He B, Wu N, Han P. 2011. Epigallocatechin-3-O-gallate (EGCG) attenuates FFAs-induced peripheral insulin resistance through AMPK pathway and insulin signaling pathway in vivo. Diabetes Res Clin Pract. 93(2):205-214.

Liu K, Zhou R, Wang B, Chen K, Shi LY, Zhu JD, Mi MT. 2013. Effect of green tea on glucose control and insulin sensitivity: a meta-analysis of 17 randomized controlled trials. Am J Clin Nutr. 98(2):340-348.

Lu H, Meng X, Yang CS. 2003. Enzymology of methylation of tea catechins and inhibition of catechol-O-methyltransferase by (-)-epigallocatechin gallate. Drug Metab Dispos. 31(5):572579. 
Martin BJ, Tan RB, Gillen JB, Percival ME, Gibala MJ. 2014. No effect of short-term green tea extract supplementation on metabolism at rest or during exercise in the fed state. Int J Sport Nutr Exerc Metab. 24(6):656-664.

Nelson KM, Weinsier RL, Long CL, Schutz Y. 1992. Prediction of resting energy expenditure from fat-free mass and fat mass. Am J Clin Nutr. 56(5):848-856.

Radermecker RP, Fayolle C, Brun JF, Bringer J, Renard E. 2013. Accuracy assessment of online glucose monitoring by a subcutaneous enzymatic glucose sensor during exercise in patients with type 1 diabetes treated by continuous subcutaneous insulin infusion. Diabetes Metab. 39(3):258262.

Randell RK, Hodgson AB, Lotito SB, Jacobs DM, Boon N, Mela DJ, Jeukendrup AE. 2013. No effect of 1 or $7 \mathrm{~d}$ of green tea extract ingestion on fat oxidation during exercise. Med Sci Sports Exerc. 45(5):883-891.

Randell RK, Hodgson AB, Lotito SB, Jacobs DM, Boon N, Mela DJ, Jeukendrup AE. 2013. No Effect of 1 or 7 Days Green Tea Extract Ingestion on Fat Oxidation during Exercise. Med Sci Sports Exerc. 45(5):883-891.

Randell RK, Hodgson AB, Lotito SB, Jacobs DM, Rowson M, Mela DJ, Jeukendrup AE. 2014. Variable duration of decaffeinated green tea extract ingestion on exercise metabolism. Med Sci Sports Exerc. 46(6):1185-1193.

Richards JC, Lonac MC, Johnson TK, Schweder MM, Bell C. 2010. Epigallocatechin-3-gallate increases maximal oxygen uptake in adult humans. Med Sci Sports Exerc. 42(4):739-744.

Santana A, Santamarina A, Souza G, Mennitti L, Okuda M, Venancio D, Seelaender M, Oller do Nascimento C, Ribeiro E, Lira F, et al. 2015. Decaffeinated green tea extract rich in 
epigallocatechin-3-gallate improves insulin resistance and metabolic profiles in normolipidic diet-but not high-fat diet-fed mice. J Nutr Biochem.

Snoussi C, Ducroc R, Hamdaoui MH, Dhaouadi K, Abaidi H, Cluzeaud F, Nazaret C, Le Gall M, Bado A. 2014. Green tea decoction improves glucose tolerance and reduces weight gain of rats fed normal and high-fat diet. J Nutr Biochem. 25(5):557-564.

Suliburska J, Bogdanski P, Szulinska M, Stepien M, Pupek-Musialik D, Jablecka A. 2012. Effects of green tea supplementation on elements, total antioxidants, lipids, and glucose values in the serum of obese patients. Biol Trace Elem Res. 149(3):315-322.

Tsuneki H, Ishizuka M, Terasawa M, Wu JB, Sasaoka T, Kimura I. 2004. Effect of green tea on blood glucose levels and serum proteomic patterns in diabetic $(\mathrm{db} / \mathrm{db})$ mice and on glucose metabolism in healthy humans. BMC Pharmacol. 4:18.

Ueda M, Nishiumi S, Nagayasu H, Fukuda I, Yoshida K, Ashida H. 2008. Epigallocatechin gallate promotes GLUT4 translocation in skeletal muscle. Biochem Biophys Res Commun. 377(1):286-290.

Venables MC, Hulston CJ, Cox HR, Jeukendrup AE. 2008. Green tea extract ingestion, fat oxidation, and glucose tolerance in healthy humans. Am J Clin Nutr. 87(3):778-784.

Venables MC, Jeukendrup AE. 2008. Endurance training and obesity: effect on substrate metabolism and insulin sensitivity. Med Sci Sports Exerc. 40(3):495-502.

Yan J, Zhao Y, Suo S, Liu Y, Zhao B. 2012. Green tea catechins ameliorate adipose insulin resistance by improving oxidative stress. Free Radic Biol Med. 52(9):1648-1657.

Zheng XX, Xu YL, Li SH, Hui R, Wu YJ, Huang XH. 2013. Effects of green tea catechins with or without caffeine on glycemic control in adults: a meta-analysis of randomized controlled trials. Am J Clin Nutr. 97(4):750-762. 
Table 1. Baseline participant characteristics

\begin{tabular}{ll}
\hline \multicolumn{1}{c}{ Variable } & \multicolumn{1}{c}{ Mean \pm SD } \\
\hline Age $(\mathrm{y})$ & $34 \pm 13$ \\
Weight $(\mathrm{kg})$ & $98 \pm 18$ \\
BMI $\left(\mathrm{kg} \cdot \mathrm{m}^{2}\right)$ & $32 \pm 5$ \\
Body fat $(\%)$ & $27 \pm 7$ \\
Vo2peak $\left(\mathrm{ml} \cdot \mathrm{kg}^{1} \cdot \mathrm{min}^{1}\right)$ & $24.2 \pm 4.2$ \\
PPO (watts) & $157 \pm 34$ \\
FPG $\left(\mathrm{mmol} \cdot \mathrm{L}^{-1}\right)$ & $6.8 \pm 1.0$ \\
\hline
\end{tabular}

BMI: body mass index, PPO: peak power output, FPG: fasting plasma glucose 
Table 2. The composition of the green tea extract used in the present study.

\begin{tabular}{lcc}
\hline \multicolumn{1}{c}{ Ingredient } & Concentration (\%) & $\begin{array}{c}\text { Supplementation dose } \\
\text { (mg/day) }\end{array}$ \\
\hline Polyphenols & 95 & 1,000 \\
Catechins & 90 & 900 \\
EGCG & 50 & 450 \\
Epigallocatechin & 20 & 180 \\
Epicatechin & 10 & 90 \\
Epicatechin gallate & 7 & 63 \\
Other Catechins & 13 & 117 \\
\hline Caffeine & $<1$ & $<9$ \\
\hline
\end{tabular}


Table 3. Effects of green tea extract (GTE) on blood measurements, pre-exercise, postexercise, and $1 \mathrm{~h}$ post-exercise in overweight, sedentary men.

\begin{tabular}{|c|c|c|c|c|c|c|}
\hline \multirow{2}{*}{ Variable } & \multicolumn{2}{|c|}{ Pre-exercise } & \multicolumn{2}{|c|}{ Post-exercise } & \multicolumn{2}{|c|}{ 1-hour post-exercise } \\
\hline & GTE & PLA & GTE & PLA & GTE & PLA \\
\hline $\begin{array}{l}\text { Glucose } \\
(\mathrm{mmol} / \mathrm{L})\end{array}$ & $6.6 \pm 0.9$ & $6.7 \pm 0.9$ & $6.7 \pm 0.7$ & $6.8 \pm 1.0$ & $8.6 \pm 1.6^{*}$ & $9.8 \pm 2.2$ \\
\hline Insulin $(\mu \mathrm{IU} / \mathrm{ml})$ & $13 \pm 8$ & $15 \pm 8$ & $10 \pm 6$ & $12 \pm 6$ & $96 \pm 59 *$ & $124 \pm 68$ \\
\hline $\begin{array}{l}\text { Free fatty acids } \\
(\mathrm{mmol} / \mathrm{L})\end{array}$ & $0.48 \pm 0.14$ & $0.51 \pm 0.11$ & $0.44 \pm 0.10$ & $0.43 \pm 0.09$ & $0.27 \pm 0.09$ & $0.29 \pm 0.14$ \\
\hline $\begin{array}{l}\text { Glycerol } \\
(\mathrm{mmol} / \mathrm{L})\end{array}$ & $0.14 \pm 0.06$ & $0.16 \pm 0.10$ & $0.17 \pm 0.06$ & $0.18 \pm 0.07$ & $0.09 \pm 0.05$ & $0.13 \pm 0.14$ \\
\hline
\end{tabular}

GTE (green tea extract), PLA (placebo). All values are means \pm SD. *denotes significance, GTE vs. PLA $(\mathrm{P} \leq 0.05)$. 


\section{Figure captions}

Figure 1. An overview of the experimental trials. Panel A depicts the overall design of the study. Subjects consumed GTE or PLA for $7 \mathrm{~d}$ periods, which were randomized in order and separated by a 2-week washout. The supplementation regimen and the timing of rest and exercise measurements are shown. "Rest" and "Exercise" indicate the timing of the resting and exercise measurements, which are detailed further in panels B and C.

Figure 2. Effects of an oral glucose beverage on 1-h post-exercise continuous glucose monitor-based glucose area under the curve (AUC) following 2 and 7 days of green tea extract (GTE) or placebo (PLA) supplementation. All values are means \pm SD. *denotes the 1h glucose AUC was significance lower with GTE vs. PLA $(\mathrm{P} \leq 0.05)$.

Figure 3. Effects of an oral glucose beverage on 1-h peak glucose and insulin post-exercise, following 2 and 7 days of green tea extract (GTE) or placebo (PLA) supplementation. Panel A depicts the blood glucose concentration $\left(\mathrm{mmol} \cdot \mathrm{L}^{-1}\right)$ pre-, post-, and 1-hr post-exercise. Panel B depicts the blood insulin concentration $\left(\mu \mathrm{IU} \cdot \mathrm{ml}^{-1}\right)$ pre-, post-, and 1-hr post-exercise. All values are means $\pm \mathrm{SD}$. *denotes postprandial rises in glucose (panel A) and insulin (panel B) were significantly lower with GTE vs. PLA $(\mathrm{P} \leq 0.05)$. 
Randomized order
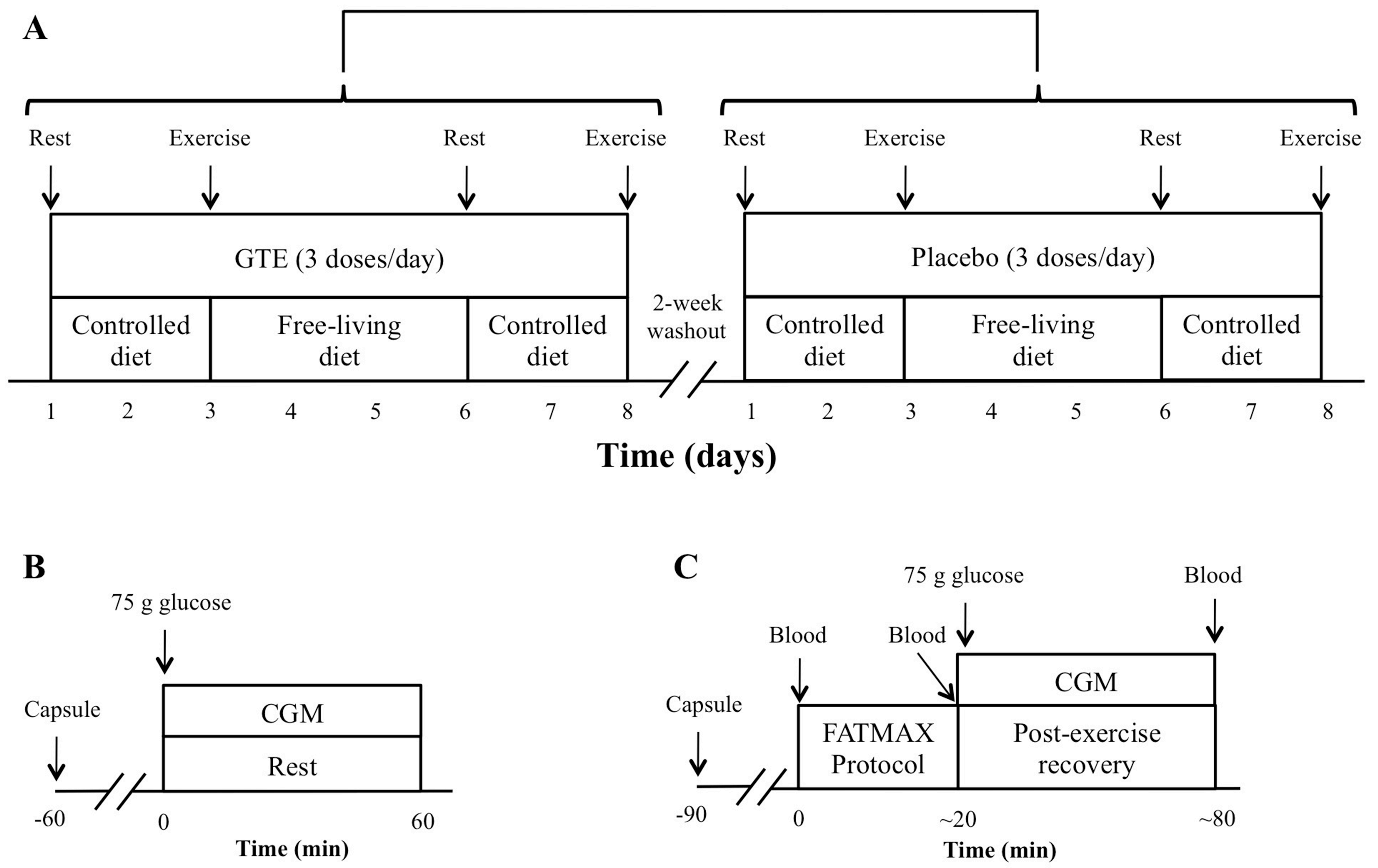


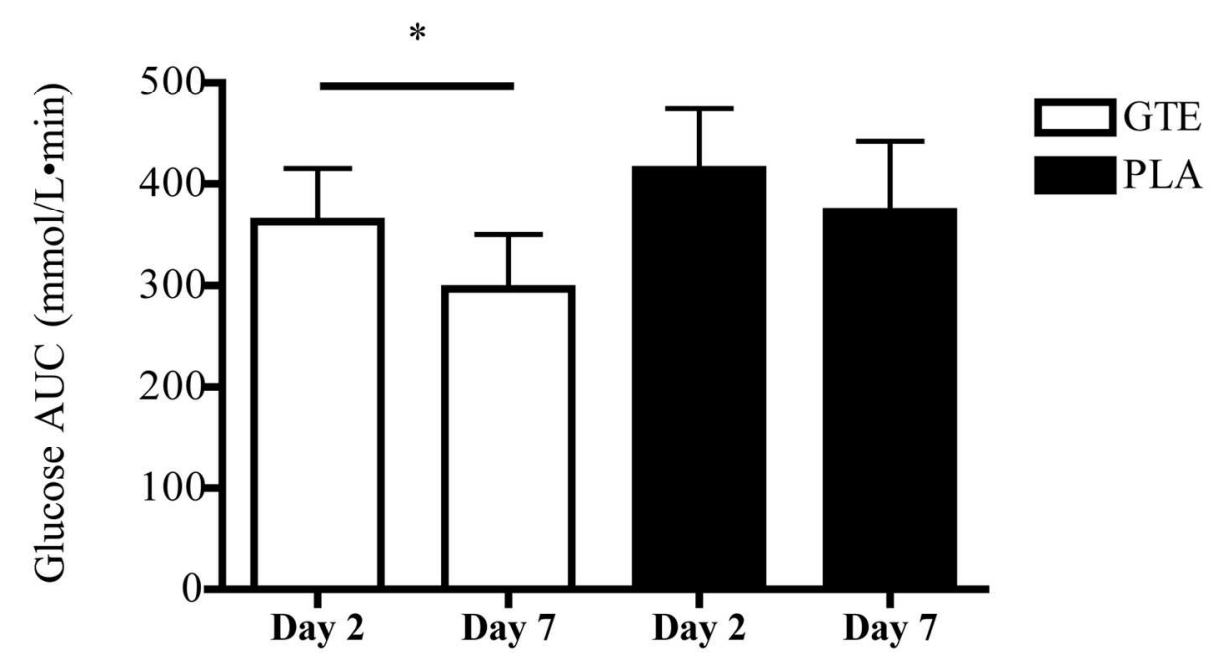

Figure 2. Effects of an oral glucose beverage on 1-h post-exercise continuous glucose monitor-based glucose area under the curve (AUC) following 2 and 7 days of green tea extract (GTE) or placebo (PLA) supplementation. GTE = open bars PLA = closed bars. All values are means \pm SD. *denotes the 1 -h glucose AUC was significance lower with GTE vs. PLA (P $\leq 0.05)$. $70 \times 38 \mathrm{~mm}(600 \times 600 \mathrm{DPI})$ 

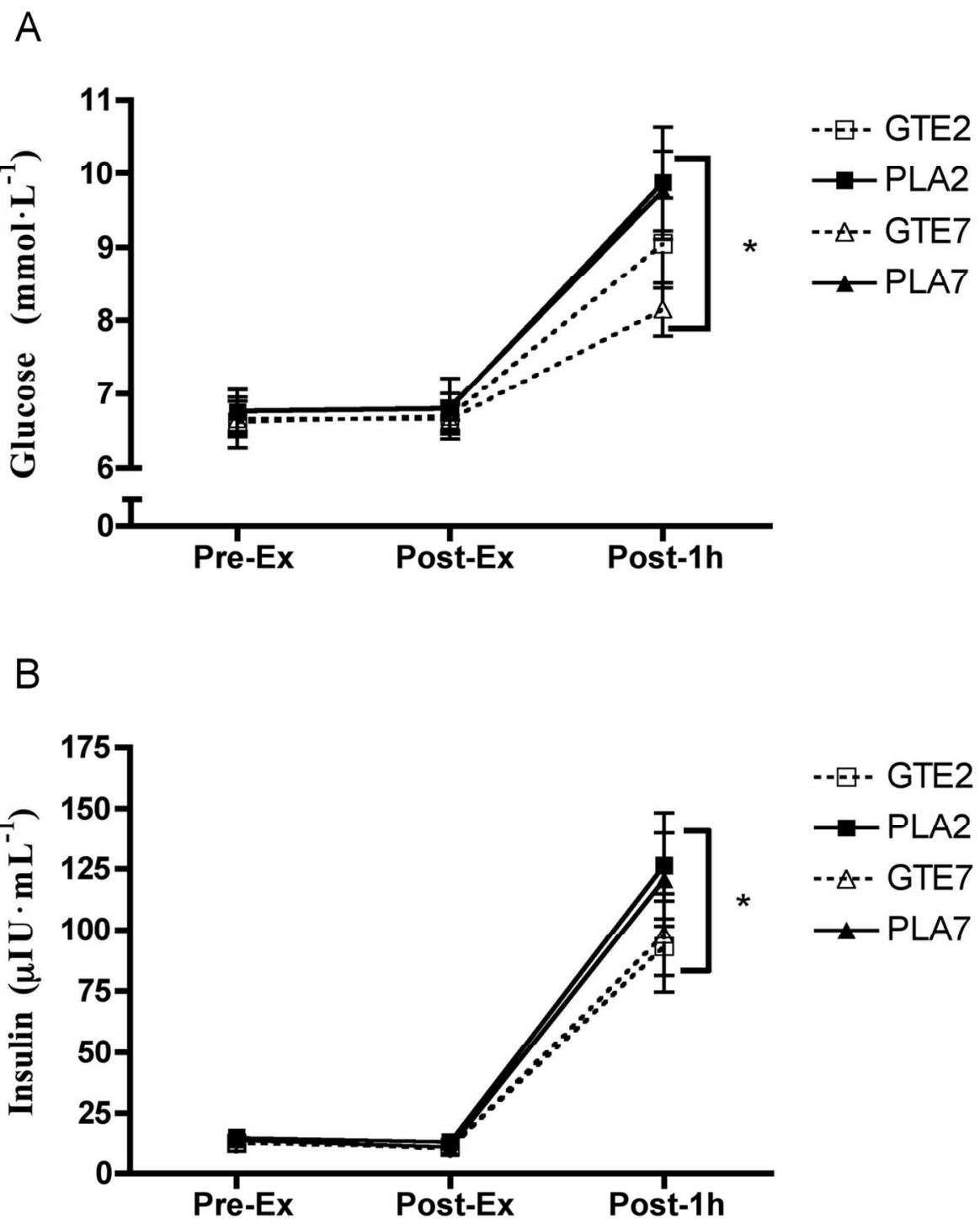

Figure 3. Effects of an oral glucose beverage on 1-h peak glucose and insulin post-exercise, following 2 and 7 days of green tea extract (GTE) or placebo (PLA) supplementation. Panel A depicts the blood glucose concentration (mmol•L-1) pre-, post-, and 1-hr post-exercise. Panel B depicts the blood insulin concentration $(\mu \mathrm{IU} \bullet \mathrm{ml}-1)$ pre-, post-, and 1 -hr post-exercise. All values are means \pm SD. $*$ denotes postprandial rises in glucose (panel A) and insulin (panel B) were significantly lower with GTE vs. PLA (P $\leq$ $0.05)$.

$121 \times 145 \mathrm{~mm}(300 \times 300 \mathrm{DPI})$ 